\title{
Complete-k-Distinguishability for Retiming and Resynthesis Equivalence Checking Without Restricting Synthesis*
}

\author{
Nikolaos Liveris \\ Northwestern University \\ Evanston, IL 60208
}

\author{
Hai Zhou \\ Fudan University, China \\ Northwestern University, USA
}

\author{
Prithviraj Banerjee \\ HP Labs \\ Palo Alto, CA 94301
}

\begin{abstract}
Iterative retiming and resynthesis is a powerful way to optimize sequential circuits but its massive adoption has been hampered by the hardness of verification. This paper tackles the problem of retiming and resynthesis equivalence checking on a pair of circuits. For this purpose we define the Complete-k-Distinguishability $(C-k-D)$ property for any natural number $k$ based on $C-1-D$. We show how the equivalence checking problem can be simplified if the circuits satisfy this property and prove that the method is complete for any number of retiming and resynthesis steps. We also provide a way to enforce $C-k-D$ on the circuits without restricting the optimization power of retiming and resynthesis or increasing their complexity. Experimental results demonstrate that enforcing $C-k-D$ property can speed up the verification process.
\end{abstract}

\section{Introduction}

A powerful optimization technique for sequential circuits is a sequence of retiming and resynthesis operations [11] (RnR sequence). Resynthesis is a combinational transformation that can be applied to blocks of logic between registers. Retiming is a sequential transformation that moves registers across gates to expose new logic blocks, giving resynthesis new opportunities for optimization. The optimization power of the RnR sequence has been discussed in many works [15]. Despite its optimization power, the RnR sequence is not widely used due to the complexity of checking sequential equivalence [7] between the initial and final design. There is a need, therefore, for efficient verification methods that preserve the optimization power of the retiming and resynthesis sequence.

Van Eijk developed an efficient method for checking sequential equivalence based on finding equivalent signals in the two circuits [14]. Jiang et. al. showed that the method is complete for sequences of retiming and resynthesis transformations with at most one resynthesis step [8]. If more than one resynthesis step has been applied and the verification procedure shows that the outputs are not equivalent, no conclusion can be drawn.

Ashar et al. [1] demonstrated that circuits with the Complete-1Distinguishability $(C-1-D)$ property can be verified with an efficient and complete method. In a C-1-D circuit, each pair of distinct states produces different output values for some input and, therefore, each state is distinguishable from any other in a single cycle. If one of the two circuits satisfies the C-1-D property, sequential equivalence checking can be reduced to combinational equivalence checking. Not all circuits satisfy C-1-D, thus the authors developed a method to enforce this property by modifying the structure of the circuit. However, a side effect of the modifications is that the optimization power of retiming and resynthesis is reduced.

A complete method to check sequential equivalence of two circuits without restrictions on the synthesis part is reachability analysis [5]. Starting with the initial state of the circuits a forward traversal of the state space can be performed to check whether a "bad state", i.e., a state where the two circuits are not equivalent, can be reached. During each iteration, the method uses the next state relation to increase the set of reachable states. In backward reachability analysis,

\footnotetext{
${ }^{*}$ Supported by NSF under CNS-0613967.
}

the process starts from the set of bad states and checks whether an initial state is reachable using the inverse of the next state relation. The number of iterations required to produce a useful answer is generally large and hard to bound. When the set of reachable states does not converge after some number of iterations, no conclusion can be drawn for the correctness of the transformations.

To improve the efficiency of reachability analysis and reduce the number of iterations without destroying completeness, a number of structural optimizations have been proposed $[9,6]$. For example, retiming can be used to reduce the number of registers before the traversal starts. These techniques can be used in conjunction with the ideas proposed in this paper.

The approach described in this paper targets the equivalence checking of a pair of circuits, one of which has been obtained from the other by a sequence of retiming and resynthesis transformations. We extend the C-1-D property to C- $k$-D, where $k \in \mathbb{N}$. A circuit fulfills the $\mathrm{C}-k$-D property if every two non-equivalent states can be distinguished within $k$ clock cycles. The contributions of this paper are the following:

1. We show how to check sequential equivalence for circuits with $\mathrm{C}-k$-D property by unrolling the product circuit a bounded number of times. This verification technique is complete; if it fails then the circuits are not RnR equivalent.

2. We provide an approach to modifying a circuit to be C- $k$-D.

3. We design a method for applying retiming and resynthesis transformations to the modified circuit, so that the optimization power is not restricted. Moreover, the complexity of retiming and resynthesis is not increased by the modification.

The modification of the circuit to enforce the C- $k$-D property is similar to target enlargement [2]. However, the method we propose is applicable even when BDD construction cannot be completed. Moreover, it is a structural transformation applied before synthesis, and it improves the verification running-time without affecting the synthesis result.

\section{Problem Formulation}

Two formalisms, namely netlists and finite state machines (FSMs), are used for representing circuits. Netlists are structural and consist of an interconnection of gates and registers. Finite state machines are behavioral and specify how the system changes its states and produces outputs responding to inputs.

A netlist is a directed graph, where the nodes correspond to elementary circuit elements, and the edges correspond to wires connecting these elements. The three basic circuit elements are primary inputs, registers, and gates. Primary input nodes have no fanins; registers have a single input. Associated with a gate $g$ on $n$-inputs $w_{1}, w_{2}, \ldots, w_{n}$ is a function from $B^{n}$ to $B$, where $B=\{0,1\}$. Some nodes are designated as primary outputs.

A Finite State Machine $(F S M)$ is a quintuple $(\Sigma, I, O, \lambda, \delta)$ where $\Sigma$ is a finite set of states, $I$ and $O$ are finite sets of inputs and outputs respectively, $\delta: \Sigma \times I \rightarrow \Sigma$ gives the next-state function, and $\lambda: \Sigma \times$ $I \rightarrow O$ the output function.

The output and next-state functions can be extended transitively to the domains $\Sigma \times I^{+} \rightarrow O^{+}$and $\Sigma \times I^{+} \rightarrow \Sigma$, respectively, where 
$A^{+}$is the set of finite non-empty sequences of elements in set $A$. We continue to use $\lambda$ and $\delta$ to denote these extended functions.

Two circuits are called compatible if they have the same set of primary inputs and primary outputs. For two compatible circuits $C_{a}$ and $C_{b}$ the product circuit $C_{x}=C_{a} \times C_{b}$ is defined. The netlist of the product circuit is built by joining the corresponding primary inputs and connecting the corresponding outputs to xor-gates forming the outputs of the product circuit. These outputs are all zero if and only if the outputs of the two circuits are equal.

A State Transition Graph (STG) of the circuit can be built for its FSM. The STG has one node for each state and its edges represent the transitions between states in one clock cycle. The longest path between any two nodes of the STG is called the diameter of the circuit.

For any circuit element $o$, we denote as $o^{(x)}$ the value of the element after $x$ clock cycles. For a predicate $\phi$ over circuit elements (e.g., registers, inputs), $\phi^{(x)}$ is obtained by replacing each circuit element by its value after $x$ clock cycles.

Retiming and resynthesis are structural operations on netlists. Retiming consists of moving a given number of registers between the inputs and outputs of each combinational node [10]. A retiming can be described mathematically by a lag function, which gives for each combinational node, the number of registers that are moved from fanout to fanin. Resynthesis restructures the netlist within register boundaries without changing its functionality. It leaves the FSM of the design unchanged.

Retiming becomes very powerful when interspersed with resynthesis of the netlist within the changing register boundaries. This is the basis for the retiming and resynthesis $(\mathrm{RnR})$ paradigm proposed in [11].

A state $s$ of a circuit is dangling, if and only if either it has no predecessor state or all its predecessor states are dangling [7]. Let $D$ represent the set of dangling states. The states in $\Sigma-D$ are called non-dangling. Two states $s_{a}$ and $s_{b}$ are equivalent, denoted as $s_{a} \approx$ $s_{b}$, if and only if

$$
\forall i \in I^{+}: \lambda_{a}\left(s_{a}, i\right)=\lambda_{b}\left(s_{b}, i\right)
$$

Two compatible circuits with a designated initial state are sequentially equivalent, if and only if their initial states are equivalent. Two compatible circuits are $R n R$ equivalent, if and only if one can be obtained from the other by a sequence of retiming and resynthesis transformations.

Problem 1 (Complete RnR Equivalence Checking) Given two circuits, check whether they are sequentially equivalent or one cannot be obtained from the other by a sequence of retiming and resynthesis operations.

For the rest of this paper we will refer to the Complete RnR Equivalence Checking Problem as RnR checking for simplicity. Obviously, this problem can be solved by model checking. However, we are interested in forcing a bound on the number of iterations to give a solution to the RnR checking problem. This bound should be forced without restricting the optimization power of the retiming and resynthesis sequence.

\section{State Characterization via Output Equivalence}

Given two compatible circuits $C_{a}$ and $C_{b}$, our first step for proving their equivalence is to build the product circuit $C_{x}$. In this section we show how to explore the structure of $C_{x}$ starting from the outputs to derive a relation between the registers of $C_{a}$ and $C_{b}$ (Section 3.1). Then we define C- $k$-D and show how it is related to the characteristic predicate of the relation between the registers (Section 3.2). This predicate is used to derive an invariant for $C_{x}$. By modifying the structure of the circuit, we can enforce C- $k$-D (Section 3.3).

\subsection{Output Equivalence}

Every output of the product circuit $C_{x}$ has the value 0 in all reachable states if and only if $C_{a}$ and $C_{b}$ are sequentially equivalent. By construction of $C_{x}$, this is equivalent to corresponding outputs $o_{a}$ and $o_{b}$ being equal in all those states. Outputs $o_{a}$ and $o_{b}$ can be expressed as functions of the registers and inputs that drive them. The function can be extracted by traversing the netlist backwards from the outputs until a register or a primary input is met. Since the inputs cannot be restricted, this is a relation over the registers of the two circuits that must hold for all input values. More precisely, let $\lambda_{a}, \lambda_{b}$ be the combinational functions that give the values of $o_{a}$ and $o_{b}$, and $R_{a}=\left\{p_{1}, p_{2}, \ldots, p_{m}\right\}, R_{b}=\left\{q_{1}, q_{2}, \ldots, q_{n}\right\}, I_{a}, I_{b}$ be the set of registers and inputs that are connected to $o_{a}$ and $o_{b}$ by a combinational path, then

$o_{a}=o_{b} \Leftrightarrow \forall i \in I: \lambda_{a}\left(p_{1}, p_{2}, \ldots, p_{m}, i\right)=\lambda_{b}\left(q_{1}, q_{2}, \ldots, q_{n}, i\right)$

where $I=I_{a} \cup I_{b}$.

We define as $\chi_{0}$ the characteristic predicate over the registers in $R_{a} \cup R_{b}$ that are connected to the outputs by a path of 0 registers, i.e.,

$$
\chi_{0} \triangleq \forall i \in I: \lambda_{a}\left(p_{1}, p_{2}, \ldots, p_{m}, i\right)=\lambda_{b}\left(q_{1}, q_{2}, \ldots, q_{n}, i\right)
$$

Predicate $\chi_{0}$ characterizes states of the product circuit that have an output value of 0 for any input.

The next state function $\delta$ is composed of a number of $\delta_{i}$ functions, each giving the value of a register in the next state as a function of the registers and the input. Function $\delta_{r_{i}}$ of register $r_{i}$ can be extracted from the circuit by traversing the signal that drives $r_{i}$ backwards until a register or an input is met. By replacing the registers of (1) with the functions giving their value in the next state, predicate $\chi_{1}$ is generated.

Predicate $\chi_{1}$ defines a relation between the register values of the two circuits that are connected by a path of exactly one register to the outputs $o_{a}$ and $o_{b}$. The predicate uses the inputs connected to those outputs by a path of 0 or 1 register to determine which states of the product circuit cause $o_{a}=o_{b}$ in the next cycle for any input, i.e.,

$$
\chi_{1} \Rightarrow o_{a}^{(1)}=o_{b}^{(1)} \Leftrightarrow \chi_{0}^{(1)}
$$

As an example, consider the two circuits shown in Figure 1. For those circuits we have

$$
\begin{aligned}
& \chi_{0}=(p 1 \vee p 2) \leftrightarrow(q 1 \wedge q 2) \\
& \chi_{1}=\forall i \in\{0,1\}:((i \wedge p 4) \vee p 3) \leftrightarrow\left(\left(i \wedge q_{4}\right) \vee \overline{q 3}\right)
\end{aligned}
$$

States $s_{a}=<p 1=0, p 2=0, p 3=0, p 4=1>$ and $s_{b}=<q 1=$ $1, q 2=0, q 3=1, q 4=0>$ satisfy $\chi_{0}$. Therefore, the output of the product circuit is zero in state $\left(s_{a}, s_{b}\right)$. However, $\left(s_{a}, s_{b}\right)$ does not satisfy $\chi_{1}$. For input $i=1$ the next state of the product circuit violates $\chi_{0}$ and the output is 1 .
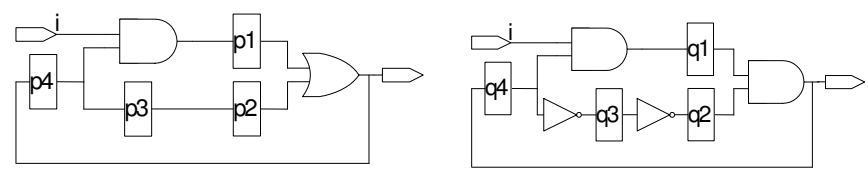

Figure 1: Two example circuits.

Similarly, by processing the circuit structure we extract $\chi_{k}$ from $\chi_{k-1}$ for any $k \in \mathbb{N}$. Moreover, we build the predicates in the same way for all outputs and for each $k \geq 0$ we take their conjunction. Therefore, the states that satisfy $\chi_{k}$ guarantee that for all xor-ed output pairs $\left(o_{a}, o_{b}\right)$ and for all input values, $o_{a}=o_{b}$ after $k$ cycles. By 
construction, it holds

$$
\chi_{k} \Rightarrow \chi_{k-1}^{(1)}
$$

for all $k \geq 1$. By the definition of $\chi_{k}$, for a pair of states satisfying $\neg \chi_{k}$ there exists an input sequence of length $k$, such that a pair of corresponding outputs are different after $k$ cycles.

States of the product circuit satisfying the conjunction

$$
\psi_{k} \triangleq \bigwedge_{i \in 0 . . k} \chi_{i}
$$

have the same output values for the first $k$ cycles. States of the two circuits that are equivalent must satisfy $\psi_{k}$ for any $k$, i.e.,

$$
\forall\left(s_{a}, s_{b}\right) \in \Sigma_{a} \times \Sigma_{b}: s_{a} \approx s_{b} \Rightarrow\left(s_{a}, s_{b}\right) \mid=\psi_{k}
$$

In the worst case the evaluation of $\psi_{k}$ for a state requires $I^{k+1}$ input values. However, by the method described above only the inputs relevant to producing the output values are processed in each of the $k+1$ cycles.

\subsection{Complete- $k$-Distinguishability}

In this section we define the C- $k$-D property and show how we can find whether a circuit satisfies it. First, we define a property for states.

Definition 1 A pair of states $s_{1}$ and $s_{2}$ of circuit $C$ are $k$-Distinguishable if and only if there exists some input sequence i of length $m \leq k$, such that $\lambda\left(s_{1}, i\right) \neq \lambda\left(s_{2}, i\right)$.

Definition 2 A circuit $C$ satisfies the $C-k-D$ property, if and only if every pair of non-equivalent states $s_{1}$ and $s_{2}$ is $k$-Distinguishable.

If we take the product $C_{x}$ of $C$ with itself, then states $s_{1}$ and $s_{2}$ are $k$ Distinguishable, if and only if $\left(s_{1}, s_{2}\right) \not \models \psi_{k-1}$. Therefore, circuit $C$ has the $\mathrm{C}-k$-D property, if and only if

$$
\forall\left(s_{1}, s_{2}\right) \in \Sigma_{x}: s_{1} \approx s_{2} \Leftrightarrow\left(s_{1}, s_{2}\right) \mid=\psi_{k-1}
$$

which follows from the definition of C- $k$-D and (3).

Note that when $k=1$ our definition of C-1-D property is more general than previous approaches [1], since we require that distinguishable be non-equivalent states, not necessarily distinct states as required by [1].

By the definition of C- $k$-D, if any circuit satisfies the C- $k$-D property, it also satisfies the C-m-D property for all $m \geq k$. Therefore, $\mathrm{C}-1-\mathrm{D}$ is the most restricted property of this class. More specifically, the circuits satisfying C-1-D are a subset of the circuits satisfying C- $k$-D for any $k \geq 1$.

Lemma 1 For every circuit $C$ there exists $k \in \mathbb{N}$ such that $C$ has the $C-k-D$ property, where $k$ is bounded from above by the diameter of $C_{x}=C \times C$.

\subsection{Convergence}

For verification purposes it is useful to have an invariant of the product circuit that implies correctness. Property $\psi_{k-1}$ implies output equivalence in the current state by construction. In this section we show that for a circuit $C$ that satisfies C- $k-\mathrm{D}, \psi_{k-1}$ is also an invariant of the product of $C$ with itself (Lemma 2). Moreover, we show how we can transform the product circuit $C_{x}=C \times C$, so that formula (4) holds for $C_{x}$. Then in Section 4 we show how we can use $\psi_{k-1}$ to check equivalence between the original and the transformed circuit.

Lemma 2 If $C$ fulfills the $C-k-D$ property, then $\psi_{k-1}$ is an invariant of $C_{x}$.
Proof: From (4) we know that $\left(s_{1}, s_{2}\right) \models \psi_{k-1}$ if and only if $s_{1}$ and $s_{2}$ are equivalent. The equivalence of these states implies that for any input $i \in I$ their next states are also equivalent, and, therefore, satisfy $\psi_{k-1}$ (Formula 3).

As we can see from the proof of Lemma 2, it is sufficient that $C_{x}$ satisfies formula (4) for $\psi_{k-1}$ to be an invariant of $C_{x}$.

In Figure 2 a Venn diagram of the state space of the product circuit can be seen. The sets of states satisfying $\psi_{k-1}, . ., \psi_{0}$ are displayed. The set of states satisfying $\psi_{m}$ is a subset of the states satisfying $\psi_{m-1}$, as $\psi_{m}=\psi_{m-1} \wedge \chi_{m}$. In order for $\psi_{k-1}$ not to be an invariant, there must be a state $s$ satisfying $\psi_{k-1}$ and having a next state $s_{1}$, such that $s_{1} \not \models \psi_{k-1}$. That means that $s_{1} \models \neg \chi_{k-1}$, since $s \models \bigwedge_{i \in 0 . k-1} \chi_{i}$ implies that all states that we can reach from $s$ in one cycle, including $s_{1}$, satisfy $\bigwedge_{i \in 0 . . k-2} \chi_{i}$, which is equivalent to $\psi_{k-2}$. This follows from (2). Moreover, from the fact that $s_{1} \models \neg \chi_{k-1} \wedge \psi_{k-2}$ we know that the path from $s$ to a state that violates output equivalence is exactly of length $k$. Each state on that path satisfies a different $\psi$ predicate and, therefore, each state on that path is distinct.

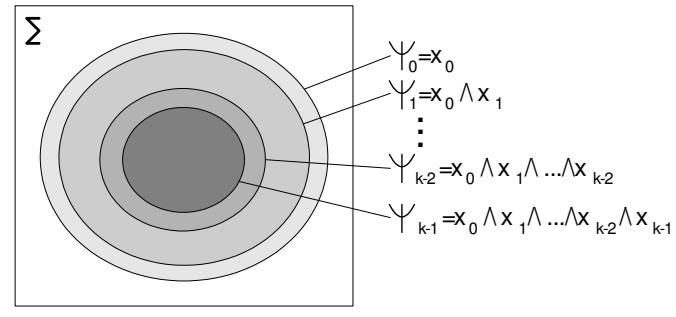

Figure 2: A Venn diagram of the states with output equivalence for the next $0 \leq m \leq k-1$ cycles.

It is possible to enforce any circuit to satisfy the C- $k$-D predicate, e.g., by the method described in [1]. However, this is not the only method. Methods to achieve this goal can be applied not only on $C$, but also on the product $C_{x}=C \times C$ of the circuit with itself. In Section 5 we provide a way to apply retiming and resynthesis without restrictions, which is independent of the method with which the C- $k$ D property was enforced.

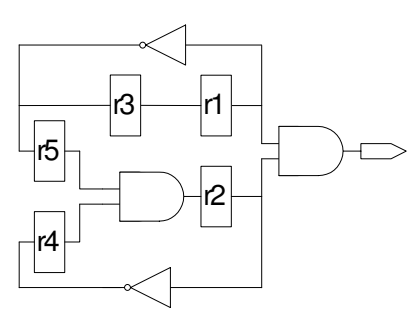

Figure 3: An example circuit.

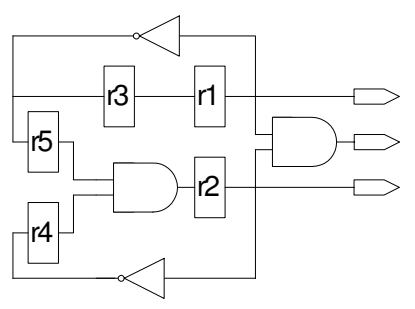

Figure 4: The circuit after the transformation.
As an example, consider the circuit of Figure 3. We take the product $C_{x}$ of that circuit with itself and denote the registers of the first and second copy as $r 1_{a}, \ldots, r 5_{a}$ and $r 1_{b}, \ldots, r 5_{b}$ respectively. For $C_{x}$ we have

$$
\begin{aligned}
& \chi_{0}=\left(r 1_{a} \wedge r 2_{a}\right) \leftrightarrow\left(r 1_{b} \wedge r 2_{b}\right) \\
& \chi_{1}=\left(r 3_{a} \wedge r 4_{a} \wedge r 5_{a}\right) \leftrightarrow\left(r 3_{b} \wedge r 4_{b} \wedge r 5_{b}\right) \\
& \chi_{2}=\left(\overline{r 1_{a}} \wedge \overline{r 2_{a}}\right) \leftrightarrow\left(\overline{r 1_{b}} \wedge \overline{r 2_{b}}\right)
\end{aligned}
$$


States $s_{a}=<r 1_{a}=0, r 2_{a}=0, r 3_{a}=0, r 4_{a}=1, r 5_{a}=0>$ and $s_{b}=<$ $r 1_{b}=1, r 2_{b}=0, r 3_{b}=0, r 4_{b}=1, r 5_{b}=0>$ satisfy $\psi_{1}=\chi_{0} \wedge \chi_{1}$, but not $\chi_{2}$. In order to enforce $\psi_{1}$ as an invariant, we create pseudooutputs and connect to them the registers with a path of 2 registers to an existing output. The set of registers satisfying this requirement is $R_{2}=\{r 1, r 2\}$. The new circuit can be seen in Figure 4. After simplifications, on the new product circuit we have

$$
\begin{aligned}
& \chi_{0}^{\prime}=\left(r 1_{a} \leftrightarrow r 1_{b}\right) \wedge\left(r 2_{a} \leftrightarrow r 2_{b}\right) \\
& \chi_{1}^{\prime}=\left(r 3_{a} \leftrightarrow r 3_{b}\right) \wedge\left(\left(r 4_{a} \wedge r 5_{a}\right) \leftrightarrow\left(r 4_{b} \wedge r 5_{b}\right)\right)
\end{aligned}
$$

States $s_{a}$ and $s_{b}$ do not satisfy $\chi_{0}^{\prime}$ and as a result they cannot satisfy $\psi_{1}^{\prime}=\chi_{0}^{\prime} \wedge \chi_{1}^{\prime}$. It can be shown that $\psi_{1}^{\prime}$ is an invariant of the modified product circuit and, therefore, the new circuit has the C-2-D property. Next, we formally describe the transformation.

Given a $k \in \mathbb{N}$ and a circuit $C$ we modify the product $C_{x}=C \times C$ of the circuit with itself, so that formula (4) holds for $C_{x}$. This has the same effect as enforcing the C- $k$-D property on $C$. Assume that $C_{x}$ is the product of the circuit with itself. We build $\psi_{k-1}$ and check whether it is an invariant. This check can be formulated as

$$
\forall\left(s_{a}, s_{b}\right) \in \Sigma_{x} \quad: \quad\left(s_{a}, s_{b}\right) \mid=\psi_{k-1} \Rightarrow\left(s_{a}, s_{b}\right) \models \chi_{k}
$$

The reason we do not need to check $\bigwedge_{j \in 0 . . k-1} \chi_{j}$ is that we know that it is satisfied by formula (2) and the definition of $\psi_{k-1}$. We assume that $k$ is chosen in such a way that the above check is tractable. The above formula cannot be false unless the circuit $C$ is not C- $k$ D. Then we chose $1 \leq m \leq k$, so that the number $\left|R_{m}\right|$ of registers connected by a path of $m$ registers to an output is minimum $(m=2$ in Figures 3, 4). We create for each register $r \in R_{m}$ a pseudo-output with the name $o_{r_{m}}$ and connect the register to the output.

Let us denote as $\chi_{0}^{\prime}, \psi_{k-1}^{\prime}$ the predicates of the circuit after the first modification. For each state $\left(s_{a}, s_{b}\right)$ with $\left(s_{a}, s_{b}\right) \not \forall \chi_{m}$ we know that there must be at least one register in $R_{m}$ that has a different value in $s_{a}$ than in $s_{b}$. By connecting those registers to outputs, when we consider $\chi_{0}^{\prime}$, we have that $\left(s_{a}, s_{b}\right) \not \neq \chi_{0}^{\prime}$. Therefore, if we start again the process of deriving $\psi_{k-1}^{\prime}$, this time $\psi_{k-1}^{\prime}$ implies $\psi_{k+m-1}$. If $\psi_{k-1}^{\prime}$ fails the invariant test, we repeat the same process after identifying another number $m^{\prime}$. Then the next predicate $\psi_{k-1}^{\prime \prime}$ tried implies $\psi_{k+m+m^{\prime}-1}$. This process continues until $\psi_{k-1}$ becomes an invariant. During this process the number of input variables for the computation of $\psi_{k-1}$ remain bounded by $k \cdot|I|$ and the number of state variables are bounded by the number $|R|$ of registers in the product circuit. Since for the number $m$ chosen in each iteration we have $m \geq 1$ and the diameter is an upper bound for $k$, eventually this process terminates. The advantage of this approach is that it is easy to apply as it does not require the BDD construction for $\psi_{n-1}$ for some $n$. For example, if for some $n \in \mathbb{N}$ the BDD construction of $\psi_{n-1}$ cannot be completed, by applying the transformation we can enforce $\psi_{l-1}$ as an invariant with $l<n$. Then $\psi_{l-1}$ could be easier to compute. The choice of $k$ for the target invariant $\psi_{k-1}$ should be made in such a way that $\psi_{k-1}$ can be computed.

The modification we described is applied to the product circuit. However, it does not prevent us from obtaining the transformed circuit by applying a sequence of retiming and resynthesis operations. In Section 5 we show how we can apply these operations without restricting their optimization power or increasing their complexity.

\section{RnR Equivalence Checking Under C- $k$-D}

In this section we show how we can check the equivalence between the original and the transformed circuit when we know that the original circuit fulfills the $\mathrm{C}-k$-D property. The check can be done by unrolling the product circuit a bounded number of times. First, we prove some properties for the non-dangling states of the original $C_{a}$ and the transformed circuit $C_{b}$. Using these properties we show that $\psi_{k-1}$ is an invariant of those circuits in the non-dangling state space, if $C_{a}$ satisfies the C- $k$-D property. Based on this result we prove the completeness of our method, i.e., if the checks fail, $C_{b}$ cannot be obtained from $C_{a}$ using retiming and resynthesis operations. Finally, we show how to check sequential equivalence between $C_{a}$ and $C_{b}$.

Lemma 3 If the original circuit $C_{a}$ is RnR equivalent to the transformed circuit $C_{b}$, then for every non-dangling state of $C_{b}$ there exists an equivalent non-dangling state in the STG of $C_{a}$.

Proof: We prove the theorem by induction. The base case, before any step of the RnR sequence, is trivial as the transformed circuit is identical to the original circuit and the STGs are isomorphic. Assume that the lemma holds after $m$ steps of the RnR sequence, we prove that it holds after the $m+1$ step has been applied.

The first case is that the $m+1$ step is a resynthesis step. Then the STG of the transformed circuit is preserved. Therefore, for every non-dangling state of $C_{b}$, there exists an equivalent non-dangling state in the STG of $C_{a}$.

The second case is that the $m+1$ is a retiming step. Let $C_{b}^{m}, C_{b}^{m+1}$ be the transformed circuits before and after the $m$ step, respectively. Retiming does not create new non-dangling states, but merges equivalent non-dangling states or splits non-dangling states to equivalent non-dangling states [7]. Consequently, for every non-dangling state in the STG of $C_{b}^{m+1}$, there exists an equivalent non-dangling state in the STG of $C_{b}^{m}$.

Lemma 4 If the original circuit $C_{a}$ has the $C-k$-D property and the transformed circuit $C_{b}$ is RnR equivalent to $C_{a}$, then $\psi_{k-1}$ is an invariant of the product circuit $C_{a \times b}=C_{a} \times C_{b}$ in the non-dangling state space.

Proof: Let us assume $C_{a}$ has the $\mathrm{C}-k-\mathrm{D}$ property and $C_{b}$ is $\mathrm{RnR}$ equivalent, but $\psi_{k-1}$ is not an invariant of $C_{a \times b}$. Then there exist $k$ distinct states $\left(s_{1}, t_{1}\right), \ldots,\left(s_{k}, t_{k}\right)$ in $C_{a \times b}$ such that

$$
\begin{aligned}
& \forall m \in 1 . . k-1 \quad: \quad \exists i \in I:\left(\delta_{a}\left(s_{m}, i\right), \delta_{b}\left(t_{m}, i\right)\right)=\left(s_{m+1}, t_{m+1}\right) \\
& \forall m \in 1 . . k-1 \quad: \quad\left(s_{m}, t_{m}\right) \models \psi_{k-m} \\
& \left(s_{k}, t_{k}\right) \models \neg \chi_{0}
\end{aligned}
$$

Since $C_{a}$ has the C- $k$-D property, there is no state $x$ such that $\left(s_{1}, x\right)=\psi_{k-1}$ and $s_{1} \not x$. This implies that $t_{1}$ is either non-equivalent to any state of $C_{a}$ or $t_{1}$ is equivalent to some state $y$ of $C_{a}$ for which $\left(s_{1}, y\right) \not \forall \psi_{k-1}$. In the first case we have that $t_{1}$ is a dangling state or $C_{b}$ is not RnR equivalent to $C_{a}$ (Lemma 3 ), which is a contradiction. Therefore, $t_{1}$ is equivalent to $y$ with $\left(s_{1}, y\right) \not \models \psi_{k-1}$. This implies that there exists $m<k-1$, such that $\left(s_{1}, y\right) \not \forall \chi_{m}$. However, then there exists an input $i \in I^{m}$ for which $\lambda_{b}\left(t_{1}, i\right)=\lambda_{a}(y, i) \neq \lambda_{a}\left(s_{1}, i\right)$. Consequently, $\left(s_{1}, t_{1}\right) \not \models \psi_{k-1}$, which is a contradiction.

Based on the lemmas above, if we are given an initial state $\left(s_{i a}, s_{i b}\right)$ for the product circuit, we can use the following method

$$
\begin{array}{rll}
\exists s_{a} \in \Sigma_{a}, \exists i \in I^{n_{d}} & : & \delta_{a}\left(s_{a}, i\right)=s_{i a} \\
\exists s_{b} \in \Sigma_{b}, \exists i \in I^{n_{d}} & : & \delta_{b}\left(s_{b}, i\right)=s_{i b} \\
\left(s_{i a}, s_{i b}\right) & \models & \psi_{k-1} \\
\forall\left(s_{a}, s_{b}\right) \in \Sigma_{a \times b}, \forall i \in I^{n_{d}} \quad: \quad & \delta\left(s_{a}, s_{b}, i\right) \models \psi_{k-1} \Rightarrow \\
& & \delta\left(s_{a}, s_{b}, i\right) \models \chi_{k}
\end{array}
$$

where $n_{d}$ is the register depth of the initial circuit. The first two formulas check that the initial states of the two circuits are nondangling. They can be posed as SAT problems. The third formula checks the initial state of the product circuit satisfies $\psi_{k-1}$. Finally, the last formula checks that $\psi_{k-1}$ is an invariant in the non-dangling state space. By considering only states reachable after $n_{d}$ cycles, the check is restricted to non-dangling states. 
If $C_{a \times b}$ satisfies formulas (5)-(8), then $C_{a}$ and $C_{b}$ are sequentially equivalent. This is because the product circuit starts from nondangling states. Then dangling states are not reachable. Moreover, $\psi_{k-1}$ is an invariant and it implies output equivalence by construction. The following theorem shows that if $C_{a}$ is a $\mathrm{C}-k$-D circuit and one of the formulas does not hold, then either $C_{a}$ and $C_{b}$ are not RnR equivalent or the initial states include dangling states.

Theorem 1 If $C_{a}$ satisfies the $C-k-D$ property, the initial state of the product circuit is non-dangling and either formula (7) or (8) does not hold, then $C_{a}$ and $C_{b}$ are not RnR equivalent.

Proof: Since the initial state of the product circuit is non-dangling, formulas (5) and (6) must hold. Then from Lemma 4, it follows that the circuits are RnR equivalent only if (8) holds. Moreover, if (7) does not hold, either dangling states are included in the initial state set (contradiction), or the circuits are not RnR equivalent.

We believe that the assumption for $\left(s_{i a}, s_{i b}\right)$ is reasonable. If $s_{i a}$ is a dangling state, then the problem of finding a corresponding state after retiming may be unsolvable.

From Theorem 1 and the discussion above, we know that if (5)(8) hold, then $C_{a}$ and $C_{b}$ are sequentially equivalent. If one of them does not hold, then $C_{b}$ cannot have been obtained from $C_{a}$ by a sequence of retiming and resynthesis transformations, i.e., $C_{b}$ is not RnR equivalent to $C_{a}$. There may be a case that $C_{b}$ is not RnR equivalent to $C_{a}$, but the two circuits are sequentially equivalent. In that case the checks for (7) and (8) may succeed or fail. In such a case a failure of the checks can point to an error of our RnR transformation implementation. A success is also a good result, because even though there may be a problem in the way the RnR transformation was implemented, the two circuits are sequentially equivalent. Ideally, in this case we would like to get both results. Our method gives only one of the results.

\section{Retiming and Resynthesis Without Restrictions}

We are given a modified product circuit $C_{x}$ that has been augmented with additional logic and outputs by a method enforcing the C- $k$-D property such as the method of Section 3.3. Assume that predicate $\psi_{k-1}$ is an invariant of $C_{x}$, and in $C_{x}$ we can distinguish the two copies of $C$, namely, $C_{1}$ and $C_{2}$, and the additional logic and outputs $C_{3}$. More specifically, each node and edge of the product circuit is colored by $\left\{c_{1}, c_{2}, c_{3}\right\}$. A $c_{3}$ edge can be driven by a gate of any color. However, a $c_{1}$ or $c_{2}$ edge can only be driven by a node of the same color. Our purpose is to apply a sequence of retiming and resynthesis transformations on $C_{2}$, the second copy of $C$, without being restricted by the additional logic. Moreover, after the transformation we want the product circuit to have $\psi_{m-1}$ as an invariant for a known $m$.

Before a resynthesis step we extract $C_{2}$ from $C_{x}$ by considering all circuit nodes and edges marked with $c_{2}$. All $c_{3}$ edges that are driven by a $c_{2}$ node are left hanging, i.e., not driven by any node, by this transformation. We express each of these edges as a function of $c_{2}$ registers and primary inputs. Then we add logic to $C_{3}$, so that the only edges hanging, i.e., not driven by a node, are the edges that would be driven by a $C_{2}$ register or a primary input. It is easy to extract the additional logic by traversing backwards a $c_{2}$ node that drives a $c_{3}$ edge until a register or a primary input is met in each path. Then this logic is replicated and added to $C_{3}$. Then we apply resynthesis on $C_{2}$. The resynthesis optimization is unrestricted as only nodes and edges of $C_{2}$ are considered. Resynthesis does not remove registers or inputs and after the step we can bring the modified version of $C_{2}$ back in $C_{x}$ by connecting $c_{2}$ nodes (registers, inputs) to the corresponding hanging $c_{3}$ edges.

Before a retiming step we extract $C_{2}$ from $C_{x}$ again. We maintain a mapping between the hanging $c_{3}$ edges and the $c_{2}$ nodes that drive those edges. We retime $C_{2}$ as an independent circuit. Retiming does not change the circuit structure, so we reconnect the modified $C_{2}$ to obtain $C_{x}$ by preserving the mapping. Nodes belonging to $C_{2}$ that drive $c_{3}$ edges may have a lag value $r$ that is different than 0 . In such a case, the number of registers on the $c_{3}$ edges need to be adjusted, so that the weights of the edges are consistent with the lag $(r-)$ values.

If for a $c_{2}$ node $v$ that drives a $c_{3}$ edge we have $r(v)<0$, then we add to the $c_{3}$ edge $-r(v)$ number of registers. These registers have been moved across $v$ from its fan-in, which are $c_{2}$ edges. Therefore, this move is based on pre-existing registers. Based on the results proved on Section 4, we know that after such a retiming move $\psi_{k-1}$ should still be an invariant of the product circuit $C_{x}$ in its nondangling state space.

In the case that $r(v)>0$ for a $c_{2}$ node $v$ driving a $c_{3}$ edge, then we remove $r(v)$ registers from the $c_{3}$ edge. If the weight of the edge becomes negative, we try to adjust the $r(u)$ value of the head $u$ of the edge, which is a $c_{3}$ node. This may cause other edges to have negative values. The paths from all these edges terminate at a single pseudo output $o$.The end result may be that we have to adjust the value of the pseudo output $o$. In that case the effect of the additional logic is moved in the past. Therefore, instead of $\psi_{k-1}$ the predicate that must be an invariant of the product circuit is $\psi_{k-1+r(o)}$. Every time we retime a pseudo output, we adjust the number $m$, for which $\psi_{m-1}$ must be an invariant of the modified product circuit. Using this number, at the end of the retiming and resynthesis sequence, we will derive $\psi_{m-1}$ and require that the checks described in Section 4 succeed, in order for the two circuits to be sequentially equivalent.

The method described in this section resembles recording the transformation history of retiming and resynthesis. In [13] a similar method is presented. There are important differences between that approach and our method. First, in [13] verification relies on synthesis to record the candidate problems to be solved. If the problems are solved successfully, then the circuits are assumed equivalent. However, it is unclear whether a bug in recording synthesis history can result in a false positive. Moreover, that technique is described for a tool that uses a specific data structure to represent a logic network, namely And-Inverter-Graphs. Our technique is general in that the data passed from synthesis to verification are in the form of a circuit. Therefore, synthesis and verification can use different data structures. In terms of efficiency, our approach creates new nodes only when required for the fan-in cones of the pseudo-outputs, while the approach in [13] stores every node created during synthesis. Moreover, it stores one node for each move of a register over a gate during a retiming operation. Because of that, restrictions on the synthesis part may become necessary for the approach in [13] to be practical.

The product circuit obtained by the method of the previous section can be used for verification purposes. However, after verification we want to extract only the optimized copy $C_{2}$ of the product circuit. It is easy to see that by taking only the nodes and edges colored by $c_{2}$, we have the optimized version. The extracted $C_{2}$ circuit is the same as the circuit obtained by applying retiming and resynthesis to $C_{1}$. The reason is that during the optimization the additional edges and nodes were not considered.

\section{Experimental Results}

We used the ABC framework [3] and VIS [4] to test our ideas. Checking formula (8) is the most difficult step of our approach, so we focus on the implementation and results for that part. With $A B C$ we implemented the check as a SAT problem on a miter. More specifically, the predicate $\psi_{k-1}$ is built as a BDD after unrolling the product circuit and applying universal quantification on the inputs. The result is appended to the circuit specifying $\chi_{k}$ using the utility of ABC that implements a BDD as a circuit of muxes. Then we check whether for any input sequence and state $\psi_{k-1} \wedge \neg \chi_{k}$ is satisfiable. The approach in [2] for building the BDD for target enlargement or the SAT approach for quantification [12] could be more efficient for check- 


\begin{tabular}{|c|c|c|c|c|c|c|c|c|}
\hline \multirow{3}{*}{ Bench } & \multicolumn{4}{|c|}{ SAT on miter with ABC } & \multicolumn{2}{|c|}{ VIS: backward traversal } & \multicolumn{2}{|c|}{ VIS: forward traversal } \\
\hline & \multicolumn{2}{|c|}{ Without Addit. Logic } & \multicolumn{2}{|c|}{ With Addit. Logic } & \multirow{2}{*}{$\begin{array}{l}\text { Without Addit. Logic } \\
\text { Exec Time (sec) }\end{array}$} & \multirow{2}{*}{$\begin{array}{l}\text { With Addit. Logic } \\
\text { Exec Time (sec) }\end{array}$} & \multirow{2}{*}{$\begin{array}{l}\text { Without Addit. Logic } \\
\text { Exec Time (sec) }\end{array}$} & \multirow{2}{*}{$\begin{array}{l}\text { With Addit. Logic } \\
\text { Exec Time (sec) }\end{array}$} \\
\hline & Exec Time (sec) & $\mathrm{k}$ & Exec Time (sec) & $\mathrm{k}$ & & & & \\
\hline s635 & 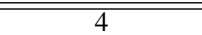 & $>4$ & 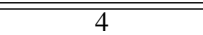 & 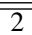 & $>>2000$ & 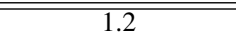 & $>>2000$ & $>>2000$ \\
\hline$s 838$ & 9.8 & $>4$ & 6.5 & 2 & 2.9 & 1.0 & 1.2 & 1.0 \\
\hline$s 938$ & 26.4 & $>4$ & 8.6 & 2 & $>2000$ & 1.9 & $>2000$ & $>2000$ \\
\hline$s 953$ & 40.2 & 3 & 3.6 & 2 & 5.1 & 2.9 & 3.3 & 2.9 \\
\hline$s 967$ & 9.6 & 3 & 5.6 & 2 & 3.1 & 5.5 & 1.7 & 2.4 \\
\hline$s 1196$ & 4.0 & 2 & 3.8 & 1 & 1.2 & 1.1 & 1.0 & 0.9 \\
\hline$s 1512$ & $>2000$ & $>4$ & 27.25 & 2 & $>2000$ & 14 & $>2000$ & $>2000$ \\
\hline$s 3271$ & $>2000$ & $>4$ & $>2000$ & 2 & $>2000$ & $>2000$ & $>2000$ & $>2000$ \\
\hline
\end{tabular}

Table 1: Running-time of different verification methods with and without additional logic. In the SAT on the miter case, $k$ is the value for which $\psi_{k}$ becomes an invariant. The computation is terminated if the running-time is greater than 2000 secs, or $k$ is greater than 4 (SAT-on-the-miter case).

ing formula (8). However, we do not expect that another method will significantly change the improvement shown in the results or the conclusions drawn by them.

Verified are a number of ISCAS benchmarks, with and without additional logic to enforce the C- $k$-D property. Each pair of circuits to verify are retiming and resynthesis equivalent. The results are reported in Table 1. The first two columns display the running time and the $k$ value of the verification process without the additional logic and output. The value $k$ is such a number that $\psi_{k}$ first becomes an invariant. The next two columns give the results with additional logic and output. Except for the additional outputs and the logic driving them the pairs of circuits are in both cases the same. The circuits with and without the additional logic were then checked with VIS. We tried both backward (columns 5 and 6) and forward traversal (columns 7 and 8) traversal with VIS. The VIS-command we used on the circuits is "seq_verify" with option "-r" for variable reordering.

In most cases the running time has been substantially reduced when additional logic and output are introduced. Examples are the cases of s635, s938, and s1512. VIS does not terminate in 2000 secs without the additional logic. However, the computation of $\psi_{k-1}$ with additional logic takes a few seconds in those cases. Enforcing $\psi_{k-1}$ can speed up the verification in VIS, as well, when it operates in backward traversal. The additional logic does not have any significant effect for the forward traversal. For the check of $\psi_{k-1}$ as an invariant, the speed up obtained by enforcing a small $k$ is significant in most cases.

From the results we conclude that by using the additional logic we can significantly speedup the checking procedure in some cases. The described procedure is based on BDDs and, therefore, does not scale well compared to SAT based procedures, e.g., [8]. However, those procedures are not proved complete for retiming and resynthesis sequences with more than one resynthesis step. We conclude that enforcing the C- $k$-D property can simplify the verification task without restricting the synthesis part.

\section{Conclusions}

In this paper we extended the property of C-1-D to C- $k$-D and we showed how we can check circuits for equivalence if one of them satisfies the C- $k$-D property. We also presented a technique to enforce the C- $k$-D property on a circuit and then apply a sequence of retiming and resynthesis transformations without restricting their optimization power or increasing their complexity. Our method provides a bound to the number of timeframes that need to processed during verification and is complete in the sense that any result provides useful information. Our experimental results show that enforcing the C- $k$-D property can speed up the verification process.

\section{References}

[1] Ashar, P., Gupta, A., And Malik, S. Using complete-1distinguishability for FSM equivalence checking. ACM Trans. Des. Au- tom. Electron. Syst. 6, 4 (2001), 569-590.

[2] Baumgartner, J., Kuehlmann, A., and Abraham, J. A. Property checking via structural analysis. In $C A V^{\prime} 02$ : Proceedings of the 14th International Conference on Computer Aided Verification (London, UK, 2002), Springer-Verlag, pp. 151-165.

[3] Berkeley Logic Synthesis and Verification Group. ABC: A System for Sequential Synthesis and Verification, Release, 70930,http://www.eecs.berkeley.edu/alanmi/abc/.

[4] Brayton, R. K., Hachtel, G. D., Sangiovanni-Vincentelli, A. L., Somenzi, F., AND ET. AL., A. A. Vis: A system for verification and synthesis. In $C A V$ '96: Proceedings of the 8th International Conference on Computer Aided Verification (London, UK, 1996), SpringerVerlag, pp. 428-432.

[5] Clarke, E. M., Grumberg, O., And Peled, D. A. Model Checking. The MIT Press, 1999.

[6] Hasteer, G., Mathur, A., And BanerJee, P. Efficient equivalence checking of multi-phase designs using retiming. Computer-Aided Design, 1998. ICCAD 98. Digest of Technical Papers. 1998 IEEE/ACM International Conference on (8-12 Nov 1998), 557-562.

[7] JiAnG, J.-H. R., AND BRAYTON, R. K. Retiming and resynthesis: A complexity perspective. Computer-Aided Design of Integrated Circuits and Systems, IEEE Transactions on 25, 12 (Dec. 2006), 2674-2686.

[8] JiAnG, J.-H. R., AND HunG, W.-L. Inductive equivalence checking under retiming and resynthesis. In ICCAD '07: Proceedings of the International Conference on Computer-Aided Design (ICCAD'07) (2007).

[9] Kuehlmann, A., AND Baumgartner, J. Transformation-based verification using generalized retiming. In $C A V$ '01: Proceedings of the International Conference on Computer Aided Verification (London, UK, 2001), Springer-Verlag, pp. 104-117.

[10] LeISERSOn, C. E., AND SAXE, J. B. Retiming synchronous circuitry. Algorithmica 6, 1 (1991), 5-35.

[11] Malik, S., Sentovich, E., Brayton, R., and SangiovanniVINCENTELLI, A. Retiming and resynthesis: optimizing sequential networks with combinational techniques. Computer-Aided Design of Integrated Circuits and Systems, IEEE Transactions on 10, 1 (Jan 1991), 74-84.

[12] McMillan, K. L. Applying sat methods in unbounded symbolic model checking. In CAV '02: Proceedings of the 14th International Conference on Computer Aided Verification (London, UK, 2002), Springer-Verlag, pp. 250-264.

[13] Mishchenko, A., AND BRAYTOn, R. K. Recording synthesis history for sequential verification. In IWLS (2008).

[14] VAN EIJK, C. A. J. Sequential equivalence checking based on structural similarities. IEEE Transactions on Computer Aided Design of Integrated Circuits and Systems 19, 7 (2000), 814-819.

[15] Zhou, H., Singhal, V., AND AzIZ, A. How powerful is retiming? In $I W L S$ (1998). 